\title{
The stellar structure of early-type galaxies: a wide-field Mitchell Spectrograph view
}

\author{
N. F. Boardman ${ }^{1} \dagger$, A. Weijmans ${ }^{1}$, R. C. E. van den Bosch ${ }^{2}$, L. Zhu ${ }^{2}$, \\ A. Yildirim ${ }^{2}$, G. van de Ven ${ }^{2}$, M. Cappellari ${ }^{3}$, P. T. de Zeeuw ${ }^{4,5}$, \\ E. Emsellem ${ }^{4}$, D. Krajnović ${ }^{6}$ and T. Naab $^{7}$ \\ ${ }^{1}$ School of Physics and Astronomy, University of St Andrews, KY16 9SS UK \\ ${ }^{2}$ Max Planck Institute for Astronomy, Königstuhl 17, D-69117 Heidelberg, Germany \\ ${ }^{3}$ Sub-department of Astrophysics, Department of Physics, University of Oxford, Denys \\ Wilkinson Building, Keble Road, Oxford OX1 3RH \\ ${ }^{4}$ European Southern Observatory, Karl-Schwarzschild-Str. 2, 85748 Garching, Germany \\ ${ }^{5}$ Sterrewacht Leiden, Leiden University, Postbus 9513, 2300 RA, Leiden, The Netherlands \\ ${ }^{6}$ Leibniz-Institut für Astrophysik Potsdam (AIP), An der Sternwarte 16, D-14482 Potsdam, \\ Germany \\ ${ }^{7}$ Max-Planck-Institut für Astrophysik, Karl-Schwarzschild-Str. 1, 85741 Garching, Germany
}

Much progress has been made in recent years towards understanding how early-type galaxies (ETGs) form and evolve. SAURON (Bacon et al. 2001) integral-field spectroscopy from the ATLAS3D survey (Cappellari et al. 2011) has suggested that less massive ETGs are linked directly to spirals, whereas the most massive objects appear to form from a series of merging and accretion events (Cappellari et al. 2013). However, the ATLAS3D data typically only extends to about one half-light radius (or effective radius, $R_{e}$ ), making it unclear if this picture is truly complete.

We observed twelve nearby ETGs using the Mitchell Spectrograph (Hill et al. 2008). We extracted stellar kinematics out to the fourth Gauss-Hermite moment, reaching $3 R_{e}$ in most cases. We found no abrupt transitions in the ETG's $\lambda_{R}$ (Emsellem et al. 2007) profiles beyond $1 R_{e}$, consistent with our ETGs having mostly passive recent histories.

We used triaxial (van den Bosch et al. 2008) Schwarzchild (1979) modelling to investigate one galaxy - NGC 3998 - in more detail (Boardman et al. 2016). We find NGC 3998 to be near-oblate, with an axis ratio $q<0.49$ at $1 R_{e}$. We obtain an I-band M/L of $4.7_{-0.45}^{+0.32}$, in good agreement with independent stellar population modelling results (Cappellari et al. 2013). Our models prefer low dark matter fractions, with a fraction of $\left(7.1_{-7.1}^{+8.1}\right) \%$ within $1 R_{e}$. Our best-fit model contains few non-rotating orbits beyond $1 R_{e}$, from which we infer that late-time accretion was not significant for this galaxy.

\section{References}

Bacon, R., Copin, Y., Monnet, G., et al. 2001, MNRAS, 326, 23

Boardman, N., Weijmans, A., van den Bosch, R. C. E. et al. 2016, MNRAS, 460, 3029

van den Bosch, R. C. E., van de Ven, G., Verolme, E. K., Cappellari, M., \& de Zeeuw, P. T. 2008, MNRAS, 385, 647

Cappellari, M., Emsellem, E., Krajnović, D., et al. 2011, MNRAS, 413, 813

Cappellari, M., McDermid, R. M., Alatalo, K., et al. 2013, MNRAS, 432, 1862

Emsellem, E., Cappellari, M., Krajnović, D., et al. 2007, MNRAS, 379, 401

Hill, G. J., MacQueen, P. J., Smith, M. P., et al. 2008, Ground-based and Airborne Instrumentation for Astronomy II, article id. 701470

Scwarzschild, M. 1979, ApJ, 232, 236

$\dagger$ E-mail: nfb@st-andrews.ac.uk 